\title{
Strong and stable photoluminescence from the semiconducting inner tubes within double walled carbon nanotubes
}

\author{
Daisuke Shimamoto, ${ }^{1}$ Hiroyuki Muramatsu, ${ }^{1}$ Takuya Hayashi, ${ }^{1}$ Yoong Ahm Kim, ${ }^{1, a)}$ \\ Morinobu Endo, ${ }^{1}$ Jin Sung Park, ${ }^{2}$ Riichiro Saito, ${ }^{2}$ Mauricio Terrones, ${ }^{3}$ and \\ Mildred S. Dresselhaus ${ }^{4}$ \\ ${ }^{1}$ Faculty of Engineering, Shinshu University, 4-17-1 Wakasato, Nagano 380-8553, Japan \\ ${ }^{2}$ Department of Physics, Tohoku University, Sendai 980-8578, Japan \\ ${ }^{3}$ Department Advanced Materials, Laboratory for Nanoscience and Nanotechnology Research (LINAN), \\ IPICYT, San Luis Potosi 78210, Mexico \\ ${ }^{4}$ Massachusetts Institute of Technology, Cambridge, Massachusetts 02139-4307, USA
}

(Received 14 October 2008; accepted 30 January 2009; published online 24 February 2009)

\begin{abstract}
We examined the optical features of single wall carbon nanotubes (SWNTs) and the inner tubes within double walled carbon nanotubes (DWNTs) having the same $(n, m)$ chirality. The brighter and more stable photoluminescence signals as well as the larger absorbance were observed for the semiconducting inner tubes within DWNTs and not for SWNTs. The outer layers of DWNTs maintain the high structural integrity of the inner tubes during both oxidative purification and strong sonication steps and are responsible in increasing the dielectric screening (due to weaker Coulomb interaction); thus leading to a redshift of the $E_{11}^{S}$ and $E_{22}^{S}$ excitonic transitions. () 2009 American Institute of Physics. [DOI: 10.1063/1.3085966]
\end{abstract}

Since the discovery of band gap photoluminescence (PL) from isolated single walled carbon nanotubes (SWNTs), ${ }^{1,2}$ PL maps have been widely utilized for determining the relative $(n, m)$ distribution of semiconducting SWNTs. ${ }^{3-5}$ The high sensitivity of PL in SWNT systems is very useful in sensing applications. ${ }^{6}$ In order to fully exploit the PL features of SWNTs in biomarkers ${ }^{7}$ and optoelectronic devices, ${ }^{8}$ strong and environmentally stable PL signatures are needed. However, SWNTs exhibit all of their constituent carbon atoms on their sidewalls and are extremely sensitive to their environments. Therefore, SWNTs reveal shifts in the transition energies $\left(E_{i i}\right)$ due to changes in their environment (i.e., dielectric constant and local strain), which is known as environmental effect. ${ }^{9-13}$ In addition, carbon nanotube defects or functional groups on the sidewalls of catalytically grown SWNTs created during oxidative purification processes, quench significantly the PL intensity.

In this study, we suggest that the inner tubes of DWNTs having thin diameter ${ }^{14}$ are preferred over SWNTs for producing photoluminescent materials. Due to their coaxial structure, the inner tubes of DWNTs are expected to possess higher structural perfection and to be more resistant toward environmental changes when compared to SWNTs; the outer tube protects effectively the inner tubes from perturbations. ${ }^{15,16}$ In order to demonstrate the above assumption, we prepared individually dispersed SWNT and DWNT suspensions using several surfactants [i.e., sodium dodecyl sulfate (SDS), sodium dodecylbenzene sulfonate (SDBS), and polyvinylpyrrolidone (PVP)] and then explored the causes for observing different optical behavior from the inner tubes of DWNTs and SWNTs having the same chirality. Even though there are a few reports on PL studies of peapod-derived DWNTs (Ref. 17) and catalytically grown

\footnotetext{
a) Author to whom correspondence should be addressed. Tel: +81-26269-5212. FAX: +81-26-269-5208. Electronic mail: yak@ endomoribu.shinshu-u.ac.jp.
}

DWNTs ${ }^{18,19}$ those studies are quite different from ours because we used highly pure crystalline DWNTs (Ref. 14) to eliminate the effect of impurities (i.e., unwanted SWNTs, amorphous carbon, and metallic impurities). In addition, the inner tubes in the present DWNTs have diameters below 0.9 $\mathrm{nm}$. We found that the strong and stable PL signal as well as the large absorbance from the inner tubes of DWNTs originates from their coaxial structures (or from the shielding effect of their outer tubes).

In this study, we have used highly purified HiPco-based SWNTs (Ref. 20) and highly pure crystalline chemical vapor deposition grown DWNTs. ${ }^{14}$ These nanotubes were individually dispersed in heavy water $\left(\mathrm{D}_{2} \mathrm{O}\right)$ with the help of SDS (1 wt \%) and SDBS $(0.5 \mathrm{wt} \%)$ under strong sonication (UP50H, $\sim 600 \mathrm{~W} / \mathrm{cm}^{2}$ ) for $1 \mathrm{~h}$ at $4{ }^{\circ} \mathrm{C}$ and subsequent ultracentrifugation (Optima Max-XP, Beckman Coulter, $240000 \mathrm{~g})$. Their supernatant $(70 \%)$ rich with isolated nanotubes, was used in our optical studies. The PVPdispersed nanotube suspensions were prepared by adding 54 $\mathrm{mg} / \mathrm{l}$ PVP to the SDBS-dispersed nanotube suspensions followed by incubation at $50{ }^{\circ} \mathrm{C}$ for $12 \mathrm{~h}^{21}$ The concentrations of isolated SWNTs and DWNTs in heavy water is adjusted to be approximately $4-5 \mathrm{mg} / \mathrm{l}$. Finally, their optical features were measured using PL (Shimadzu, NIR-PL system) and an optical absorption spectrometer (Shimadzu, SolidSpec-3700).

When recording the PL maps for the inner tubes of DWNTs and for SWNTs having the same $(n, m)$ chirality [Fig. 1(a)], the tubes were individually dispersed in $\mathrm{D}_{2} \mathrm{O}$ with the help of SDBS. We have used slit widths of $10 \mathrm{~nm}$ and scan steps of $0.5 \mathrm{~nm}$ for obtaining excitation and emission spectra, respectively. Each peak in the PL map corresponds to an excitation involving the second excitonic transition $E_{22}^{S}$ of the semiconducting inner tubes of the DWNTs and of semiconducting SWNTs and the emission from the corresponding first excitonic transition $E_{11}^{S}$ of semiconducting inner tubes of DWNTs and of semiconducting SWNTs. 

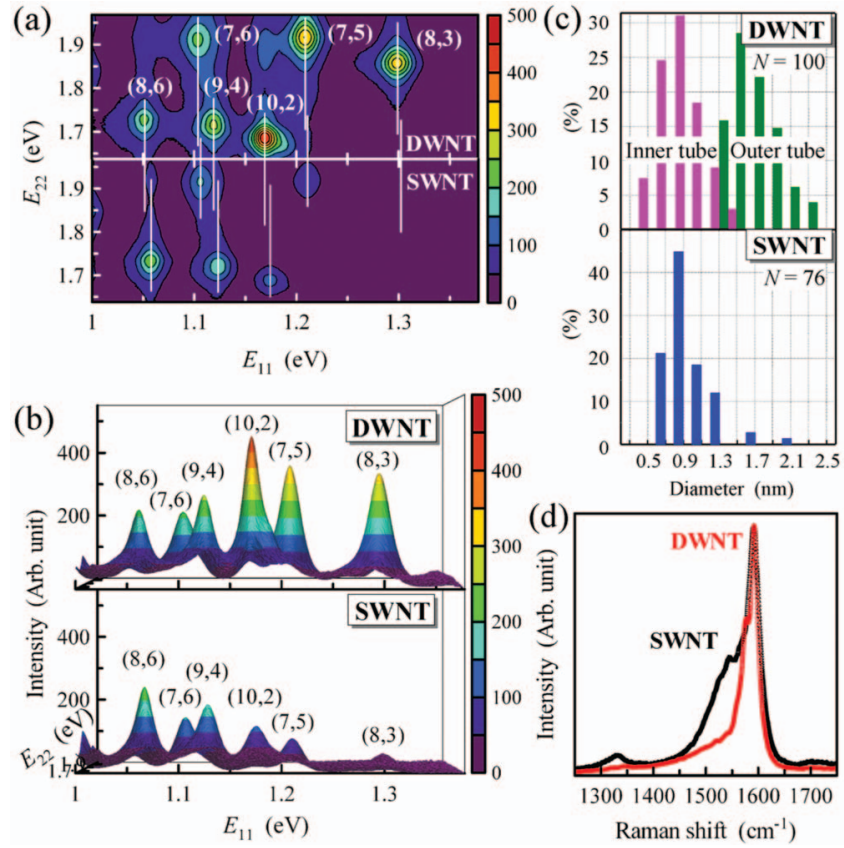

FIG. 1. (Color) The PL maps (a) and intensities (b) of the SDBS-dispersed DWNT and SWNT suspensions in $\mathrm{D}_{2} \mathrm{O}$ (c) the diameter distributions and (d) Raman spectra of DWNTs and SWNTs.

From these PL maps, we observed a redshift of both the $E_{11}$ and $E_{22}$ from the semiconducting inner tubes of the DWNTs as compared to SWNTs. This energy difference $\left(\Delta E_{i i}\right.$ $\left.=E_{i i}^{\mathrm{DWNT}}-E_{i i}^{\mathrm{SWNT}}\right)$ for each individual system is summarized in Table I. The average difference of $E_{11}^{S}$ is $-4 \mathrm{meV}$ while the average difference of $E_{22}^{S}$ is $-4 \mathrm{meV}$. In addition, there is no distinctive diameter (chirality) dependence of this difference. According to important studies on the environmental effect of PL in SWNTs, ${ }^{9-13}$ the dielectric screening effects in DWNTs should be considered because the inner tubes are surrounded by the semiconducting outer tubes with an interlayer spacing of approximately $0.31 \mathrm{~nm},{ }^{22}$ which is inaccessible by water molecules. More specifically, the inaccessibility of water molecules into the semiconducting inner tubes in DWNTs decreases the Coulomb interactions relative to SWNTs, resulting in a redshift of the $E_{11}^{S}$ and $E_{22}^{S}$ transitions. Noticeably, we found that the PL from the semiconducting inner tubes in DWNTs is more intense than that from SWNTs, except for the $(8,6)$ tube [see Fig. 1(b) and Table I]. When considering coaxial structure and one possible lumi- nescent configuration (semiconducting inner tube at semiconducting outer tube) of DWNTs, the apparent number of the semiconducting inner tubes of the DWNTs contributing to the strong PL is theoretically expected to be one-fourth of SWNTs according to the experimentally determined same concentration and similar diameter distributions [Fig. 1(c)] for both samples. This unexpected bright PL from the inner tubes of the DWNTs can be explained by the small difference in the $E_{11}$ of the inner tube and the $E_{22}$ of the outer tube that enhances the energy transfer from the outer to the inner tubes, in which the possible $(n, m)$ values and the $E_{i i}$ values for the outer tubes are listed in Table I. For smaller diameter tubes [e.g., $(8,3),(7,5)$, and $(10,2)], E_{22}^{S}$ (outer) $-E_{11}^{S}$ (inner) is much smaller than that for the larger diameter species [i.e., $(7,6),(9,4),(8,6)]$. Thus, we expect a stronger energy transfer from $E_{22}^{S}$ (outer) to $E_{11}^{S}$ (inner) for the smaller diameter tubes. Furthermore, the high structural integrity (low fraction of defects) of the inner tubes of the DWNTs [see Fig. 1(d)] is partially contributing to the strong PL because the outer tubes effectively shielded the inner tubes from severe oxidation attack in the high-temperature oxidative purification step $^{14}$ and from the high shear force in the strong sonication process.

Thus, in order to explain the shielding effect of the outer tubes in DWNTs, we compared the difference $\left(\Delta E_{i i}\right)$ of inner tubes in DWNTs and SWNTs by changing surfactants from SDS to SDBS [Fig. 2(a)] and SDBS to PVP [Fig. 2(b)]. We found that the difference of $E_{22}^{S}$ in SWNTs exhibited a chiral angle dependence which can be explained by the matriximposed stress. ${ }^{12}$ However, there is no distinctive change in the difference between the $E_{11}^{S}$ and $E_{22}^{S}$ from the inner tubes of the DWNTs. This result also confirms the effective protective function of the outer tubes in DWNTs against environmental forces. Therefore, in order to explain small variations of $E_{i i}$ (from SDS to SDBS) [Fig. 2(a)], we performed semiempirical molecular orbital calculation using the PM6 method with MOPAC2007 (Refs. 23 and 24) in order to study the change in the charge density distribution of DWNTs (Ref. 25) and SWNTs by attaching a single SDBS molecule to the sidewall [Fig. 2(c)]. Both types of tubes clearly show charge polarization and both also share charges between their outmost shell and the top of the hydrophilic group in SDBS. However, charge sharing in SWNTs is quite different from DWNTs because the Coulomb interactions are inversely proportional to the interparticle distance. For DWNTs, charge

TABLE I. Experimental transition energies and PL intensities of SDBS-wrapped DWNTs and SWNTs.

\begin{tabular}{|c|c|c|c|c|c|c|c|c|c|c|c|c|c|c|}
\hline \multirow[b]{2}{*}{$(n, m)$} & \multirow[b]{2}{*}{$\begin{array}{l}2 n+m, \\
\text { type I/II }\end{array}$} & \multirow[b]{2}{*}{$\begin{array}{c}d_{t} \\
(\mathrm{~nm})\end{array}$} & \multirow[b]{2}{*}{$\theta$} & \multicolumn{3}{|c|}{ SWNT } & \multicolumn{3}{|c|}{ Inner-DWNT } & \multicolumn{2}{|c|}{ DWNT-SWNT } & \multicolumn{3}{|c|}{ Possible outer-DWNT } \\
\hline & & & & $\begin{array}{l}E_{11} \\
(\mathrm{eV})\end{array}$ & $\begin{array}{l}E_{22} \\
(\mathrm{eV})\end{array}$ & $\mathrm{I}_{\mathrm{PL}}{ }^{\mathrm{a}}$ & $\begin{array}{l}E_{11} \\
(\mathrm{eV})\end{array}$ & $\begin{array}{l}E_{22} \\
(\mathrm{eV})\end{array}$ & $\mathrm{I}_{\mathrm{PL}}{ }^{\mathrm{a}}$ & $\begin{array}{l}\Delta E_{11}{ }^{\mathrm{b}} \\
(\mathrm{meV})\end{array}$ & $\begin{array}{l}\Delta E_{22}{ }^{\mathrm{b}} \\
(\mathrm{meV})\end{array}$ & $(n, m)$ & $\begin{array}{l}\Delta d_{t}{ }^{\mathrm{c}} \\
(\mathrm{nm})\end{array}$ & $\begin{array}{c}\Delta E^{\mathrm{d}} \\
(\mathrm{meV})\end{array}$ \\
\hline$(8,3)$ & $19, \mathrm{I}$ & 0.77 & 15.3 & 1.302 & 1.860 & 50 & 1.298 & 1.859 & 331 & -4 & -2 & $(14,6)$ & 0.62 & 29 \\
\hline$(7,5)$ & $19, \mathrm{I}$ & 0.82 & 24.5 & 1.211 & 1.919 & 96 & 1.209 & 1.916 & 351 & -2 & -3 & $(18,1)$ & 0.61 & 18 \\
\hline$(10,2)$ & $22, \mathrm{I}$ & 0.87 & 9.0 & 1.174 & 1.689 & 120 & 1.169 & 1.685 & 450 & -5 & -4 & $(15,7)$ & 0.63 & 59 \\
\hline$(7,6)$ & 20, II & 0.88 & 27.5 & 1.106 & 1.916 & 157 & 1.103 & 1.911 & 219 & -3 & -5 & $(17,4)$ & 0.62 & 192 \\
\hline$(9,4)$ & $22, \mathrm{I}$ & 0.90 & 17.5 & 1.123 & 1.718 & 189 & 1.119 & 1.714 & 267 & -4 & -4 & $(16,6)$ & 0.62 & 140 \\
\hline$(8,6)$ & $22, \mathrm{I}$ & 0.95 & 25.3 & 1.057 & 1.734 & 242 & 1.051 & 1.728 & 221 & -6 & -6 & $(15,8)$ & 0.62 & 173 \\
\hline
\end{tabular}

${ }^{\mathrm{a}} \mathrm{I}_{\mathrm{PL}}$ is experimentally obtained from 3D photoluminescence maps [Fig. 1(b)].

${ }^{\mathrm{b}}$ The energy differences $\left(\Delta E_{i i}=E_{i i}^{\mathrm{DWNT}}-E_{i i}^{\mathrm{SWNT}}\right)$ are calculated.

${ }^{\mathrm{c}}$ The diameter differences between inner and outer tubes are calculated.

${ }^{\mathrm{d}}$ The energy differences $\left[\Delta E=E_{22}^{S}(\right.$ outer $)-E_{11}^{S}$ (inner) $]$ are calculated. 

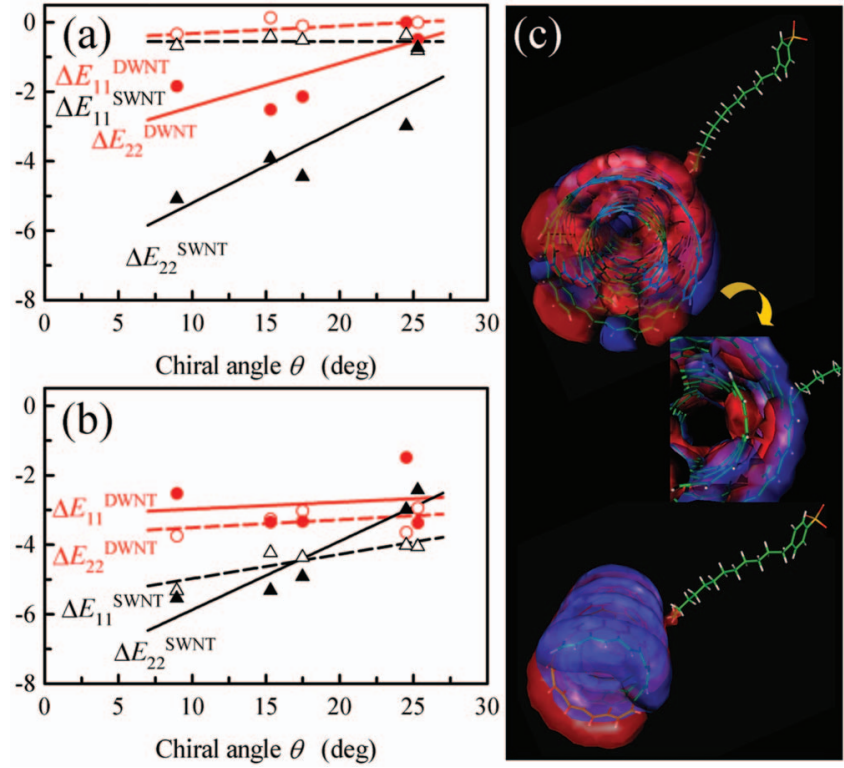

FIG. 2. (Color) (a) The difference of $E_{11}^{S}$ and $E_{22}^{S}$ transitions by changing surfactants from (a) SDS to SDBS and (b) SDBS to PVP as a function of chiral angle. (c) The charge density distributions of the (10,2) SWNT and $(10,2)$ at $(16,6)$ DWNT by attaching a SDBS molecule. The inset of (c) is an enlarged image of inner tube of the DWNT near the point to the adjacent SDBS.

polarization occurs mainly on the outer tube and thus the polarization of the inner tube of the DWNTs is significantly weaker than for SWNTs [the inset of Fig. 2(b)]. As a result, the electronic structure of SWNTs is largely perturbed as compared to that for the inner tube of the DWNT.

Finally, we have studied the concentration effect for a series of seven DWNT and SWNT mixed solutions using an optical absorption spectrometer (Fig. 3). It is noteworthy that well-resolved and sharp optical absorption peaks from the inner tubes of the DWNTs (Ref. 26) are six times more intense than those from SWNTs, possibly due to the intrinsic high molar extinction coefficient of the inner tubes. Note that the inner tubes of the DWNTs have also a higher structural integrity when compared to SWNTs. In addition, the absorption peaks clearly move toward low energies with increasing concentration of DWNTs. The lower energy for the $E_{i i}$ from the inner tubes of the DWNTs originates from their intrinsic coaxial structure.

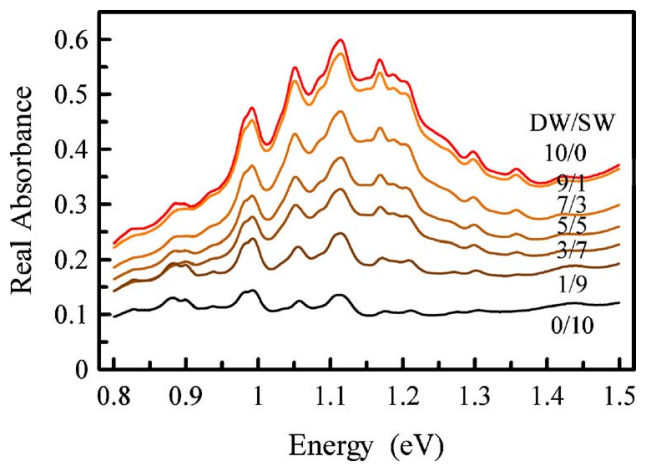

FIG. 3. (Color online) The variation in the optical absorption spectra as a function of the SDBS-dispersed DWNT/SWNT concentrations in $\mathrm{D}_{2} \mathrm{O}$.
In summary, we envisage that DWNTs will in the future replace SWNTs in biomarker and optoelectronics applications due to their strong and stable photoluminescence properties of their environmentally shielded inner tubes.

We (M.E., R.S., and Y.A.K.) acknowledge the support from the CLUSTER (second stage) and the MEXT grants (Nos. 19002007, 20241023, 16076201, and 20510096). M.S.D. acknowledges support from NSF/DMR grant (No. 07-04197). M.T. acknowledges several grants (Nos. 56787, 45772, 41464, and 2004-01-013).

${ }^{1}$ M. J. O'Connell, S. M. Bachilo, C. B. Huffman, V. C. Moore, M. S. Strano, E. H. Haroz, K. L. Rialon, J. Boul, W. H. Noon, C. Kittrell, J. Ma, R. H. Hauge, R. B. Weisman, and R. E. Smalley, Science 297, 593 (2002).

${ }^{2}$ S. M. Bachilo, M. S. Strano, C. Kittrell, R. H. Hauge, R. E. Smalley, and R. B. Weisman, Science 298, 2361 (2002).

${ }^{3}$ S. Lebedkin, F. Hennrich, T. Skipa, and M. M. Kappes, J. Phys. Chem. B 107, 1949 (2003).

${ }^{4}$ Y. Miyauchi, S. Chiashi, Y. Murakami, Y. Hayashida, and S. Maruyama, Chem. Phys. Lett. 387, 198 (2004).

${ }^{5}$ T. Okazaki, T. Saito, K. Matsuura, S. Ohshima, M. Yumura, Y. Oyama, R. Saito, and S. Iijima, Chem. Phys. Lett. 420, 286 (2006).

${ }^{6}$ D. A. Heller, E. S. Jeng, T.-K. Yeung, B. M. Martinez, A. E. Moll, J. B. Gastala, and M. S. Strano, Science 311, 508 (2006).

${ }^{7}$ D. A. Heller, S. Baik, T. E. Eurell, and M. S. Strano, Adv. Mater. (Weinheim, Ger.) 17, 2793 (2005).

${ }^{8}$ M. E. Itkis, F. Boronndics, A. Yu, and R. C. Haddon, Science 312, 413 (2006).

${ }^{9}$ J. Lefebvre, J. M. Fraser, Y. Homma, and P. Finnie, Appl. Phys. A: Mater. Sci. Process. 78, 1107 (2004).

${ }^{10}$ P. Finnie, Y. Homma, and J. Lefebvre, Phys. Rev. Lett. 94, 247401 (2005)

${ }^{11}$ Y. Ohno, S. Iwasaki, Y. Murakami, S. Kishimoto, S. Maruyama, and T. Mizutani, Phys. Rev. B 73, 235427 (2006).

${ }^{12}$ K. Arnold, S. Lebedkin, O. Kowski, F. Hennrich, and M. M. Kappes, Nano Lett. 4, 2349 (2004).

${ }^{13}$ Y. Miyauchi, R. Saito, K. Sato, Y. Ohno, S. Iwasaki, T. Mizutani, J. Jiang, and S. Maruyama, Chem. Phys. Lett. 442, 394 (2007).

${ }^{14}$ M. Endo, H. Muramatsu, T. Hayashi, Y. A. Kim, M. Terrones, and M. S Dresselhaus, Nature (London) 433, 476 (2005).

${ }^{15}$ H. Muramatsu, Y. A. Kim, T. Hayashi, M. Endo, A. Yonemoto, H. Arikai, F. Okino, and H. Touhara, Chem. Commun. (Cambridge) 2005, 2002.

${ }^{16}$ Y. A. Kim, H. Muramatsu, K. C. Park, D. Shimamoto, Y. C. Jung, J. H. Kim, T. Hayashi, Y. Saito, M. Endo, M. Terrones, and M. S. Dresselhaus, Appl. Phys. Lett. 93, 051901 (2008).

${ }^{17}$ T. Okazaki, S. Bandow, G. Tamura, Y. Fujita, K. Iakoubovskii, S. Kazaoui, N. Minami, T. Saito, K. Suenaga, and S. Iijima, Phys. Rev. B 74, 153404 (2006).

${ }^{18}$ T. Hertel, A. Hagen, V. Talaev, K. Arnold, F. Henrich, M. Kappes, S. Rosenthal, J. McBride, H. Ulbricht, and E. Flahaut, Nano Lett. 5, 511 (2005).

${ }^{19}$ N. Kishi, S. Kikuchi, P. Ramesh, T. Sugai, Y. Watanabe, and H. Shinohara, J. Phys. Chem. B 110, 24816 (2006)

${ }^{20}$ M. J. Bronikowski, P. A. Willis, D. T. Colbert, K. A. Smith, and R. E. Smalley, J. Vac. Sci. Technol. A 19, 1800 (2001).

${ }^{21}$ M. J. O'Connell, P. Boul, L. M. Ericson, C. Huffman, Y. Wang, E. Haroz, C. Kuper, J. Tour, K. D. Ausman, and R. E. Smalley, Chem. Phys. Lett. 342, 265 (2001).

${ }^{22}$ F. Villalpando-Paez, H. Son, D. Nezich, Y. P. Hsieh, J. Kong, Y. A. Kim, D. Shimamoto, H. Muramatsu, T. Hayashi, M. Endo, M. Terrones, and M. S. Dresselhaus, Nano Lett. 8, 3879 (2008).

${ }^{23}$ J. J. P. Stewart, MOPAC2007, Stewart Computational Chemistry, Colorado Springs, CO, USA, http://openmopac.net.

${ }^{24}$ J. J. P. Stewart, J. Mol. Model. 13, 1173 (2007).

${ }^{25}$ A. G. Souza Filho, M. Endo, H. Muramatsu, T. Hayashi, Y. A. Kim, E. B. Barros, N. Akuzawa, Ge. G. Samsonidze, R. Saito, and M. S. Dresselhaus, Phys. Rev. B 73, 235413 (2006).

${ }^{26}$ J. H. Kim, M. Kataoka, Y. A. Kim, D. Shimamoto, H. Muramatsu, T. Hayashi, M. Endo, M. Terrones, and M. S. Dresselhaus, Appl. Phys. Lett. 93, 223107 (2008) 